\title{
VR Mobile Acrophobia Treatment
}

\author{
Ahmed A. H. Alkurdi \\ Department of Computer Science and Information Technology, College of Computer Science \& Information \\ Technology, Nawroz University, Duhok, Iraq
}

\begin{abstract}
Virtual Reality applications have significantly developed over the past few years. The large computing power nowadays has empowered building applications with high quality graphics that can emulate the real world. This advancement has contributed to the psychiatric department in the process of anxiety treatment.

Experiencing fear in dangerous situations is natural. Usually people are afraid of predators, insects and different hazardous environments. However, certain people have anxiety problems from situations, living creatures, places or things were it is natural to others. The term phobia refers to excessive irrational fear and anxiety in natural situations. Phobias have disrupted people's life in many ways. Acrophobia or "Fear of Heights" is were patients experience irrational fear of heights.

Virtual Reality has had a great contribution to phobia treatment. As patients can be exposed to their fears in a gradual manner. In this paper, a VR environment was developed to help psychiatrists in Acrophobia diagnosed patients' treatment. This article focuses on VR environment development and its limitations on a mobile platform. Since mobile devices processing power is much less than VR sets which are usually used. The modeling and texturing limitations imposed by mobile hardware greatly restrict detail.
\end{abstract}

KEYWORDS: Acrophobia Treatment, Exposure Therapy, Virtual reality, Mobile application, Feasible.

\section{INTRODUCTION}

Virtual reality applications have grown interest in the field of entertainment and psychology. Psychologically, virtual reality applications aids in the exposure therapy used by psychiatrics for specific phobia treatment. Psychiatrics take advantage of virtual reality to immense their patients in feared situations (Parab, Pawar, \& Chaudhari, 2016). In 1987, the American Psychiatric Association reported that lifetime phobia occurrence was at $10.4 \%$, while an epidemiology study reported $11.3 \%$. more precisely $3.5 \%$ of patients have fear of flying, while $5.3 \%$ had fear of heights (Krijn, 2006). Moreover, a study conducted in Dutch land foresaw that over $10 \%$ of Dutch population will be diagnosed with some sort of phobia during their lifetime (Maatjes, 2005).

Exposure therapy has been found effective to patients with specific phobias. In traditional exposure therapy, patients are set in their feared situation for 3 hour sessions.

Academic Journal of Nawroz University (AJNU)

Volume 6, No 3(2017), 5 pages

Received 1 May 2017; Accepted 29 August 2017

Regular research paper: Published 30 August 2017

Corresponding author's e-mail: ahmed.ala89@nawroz.edu.krd

Copyright (C2017 Ahmed A. H. Alkurdi

This is an open access article distributed under the Creative

Commons Attribution License.
However, this method is costly and might even be dangerous because it is hard to maintain stimuli for the patients' specific phobias. Also patients are reluctant to seek sessions because of their anxiety problems. Another method is virtual reality exposure therapy, which is more cost effective, safe and easy to maintain (Miloff, Lindner, Hamilton, Reuterskiold, Andersson, \& Carlbring, 2016).

This research attempts to create a more feasible, cost effective method of virtual reality exposure treatment. The tools usually used for the VR exposure therapy are specific VR headsets with high end computers. Which restricts availability to most psychologists at their private clinics. This paper highlights the ability of building such applications on mobile devices that can be used with VR Headsets, such as the Samsung Gear VR by Oculus, which are practical, available and more cost effective. Furthermore, this also suggests the possibility of selfadministered exposure sessions for patients at the comfort of their homes. Which of course must be done after psychiatric consultation.

\section{Design}

The modeling has been done using Maya3d modeling software to produce a replica of the San Francisco Golden Gate bridge. Maya3d is a modeling and animation rendering software used for environments, virtual reality and character creation (Autodesk Inc., 2016) (Technologies) (Unity Technologies, 2017) (Unity 
Technologies, 2017).

\subsection{Modeling}

The modeling has been done several times to accustom the VR performance criteria. The limitation imposed by the VR hardware limit the number of polygons used to model the bridge.

The tower of the bridge has been modeled using a single polygon were normally it would incorporate several polygons see (figure 2.1). Hence the model made up of a single polygon which is the base see (figure 2.2). afterwards the base structure is extruded using the modeling toolkit integrated with Maya3d across specific faces with edge loops to introduce the complete tower bridge.

Modeling of the other parts of the bridge continues likewise. The bridge is modeled using two bridges, a set of polygon cylinders and the bridge deck for the street. The polygon cylinders are used to model the cables holding the bridge from the tower. Which are bent using the animation tools see (figure 2.3).
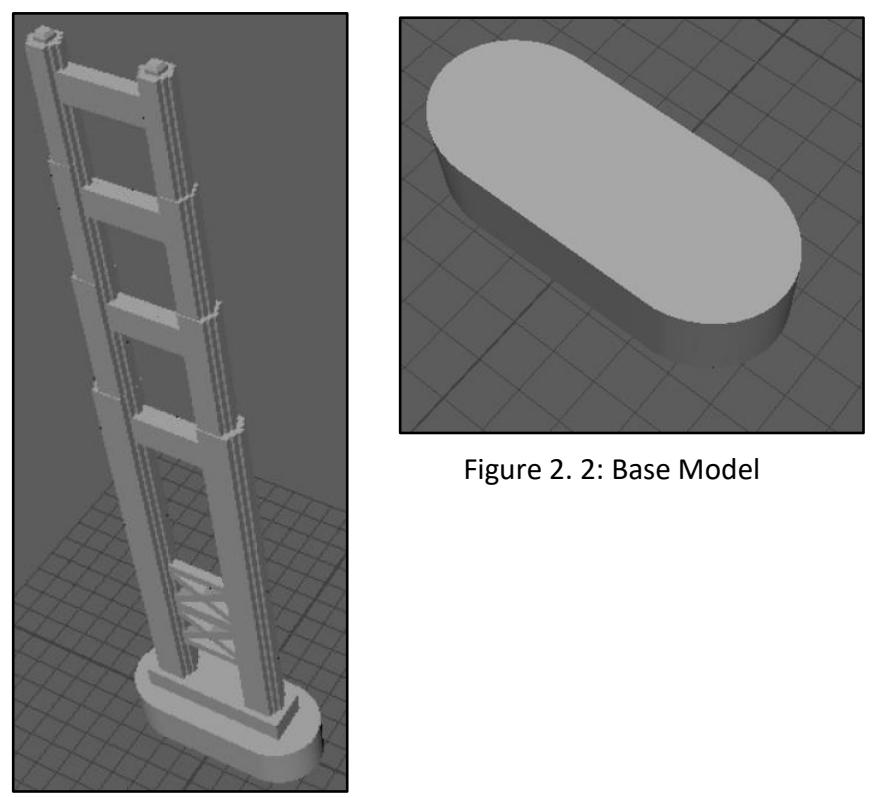

Figure 2. 2: Base Model

Figure 2. 3: Tower Model

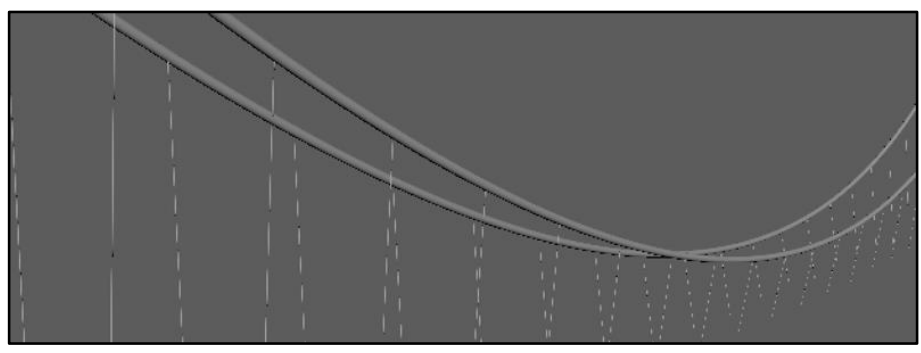

Figure 2. 5: Cables Model

Furthermore. The bridge deck is combined of a few number of polygon cubes accommodating the restrictions posed by the VR hardware. These models are then combined to produce the final bridge model see (figure 2.4).

The application also incorporates an elevator on one side of one tower to introduce gradual height to patents. The elevator is made from five polygons which are combined into two. The polygons compose the elevator body, and the elevator door, which must be kept separated for animation purposes. See (figure 2.5). The elevator itself also must be kept separated from the bridge also for animation purposes. as the only separate polygons can be animated separately.

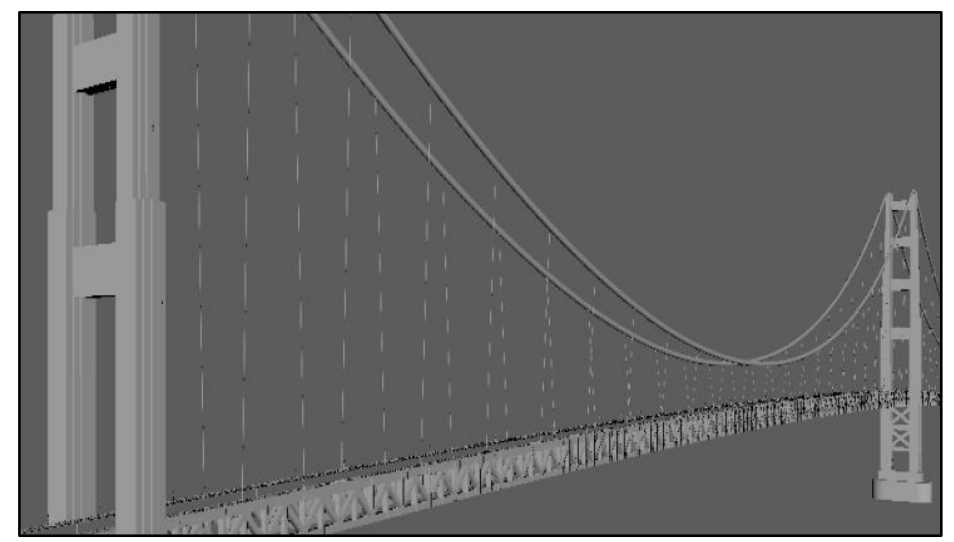

Figure 2. 1: Bridge Model

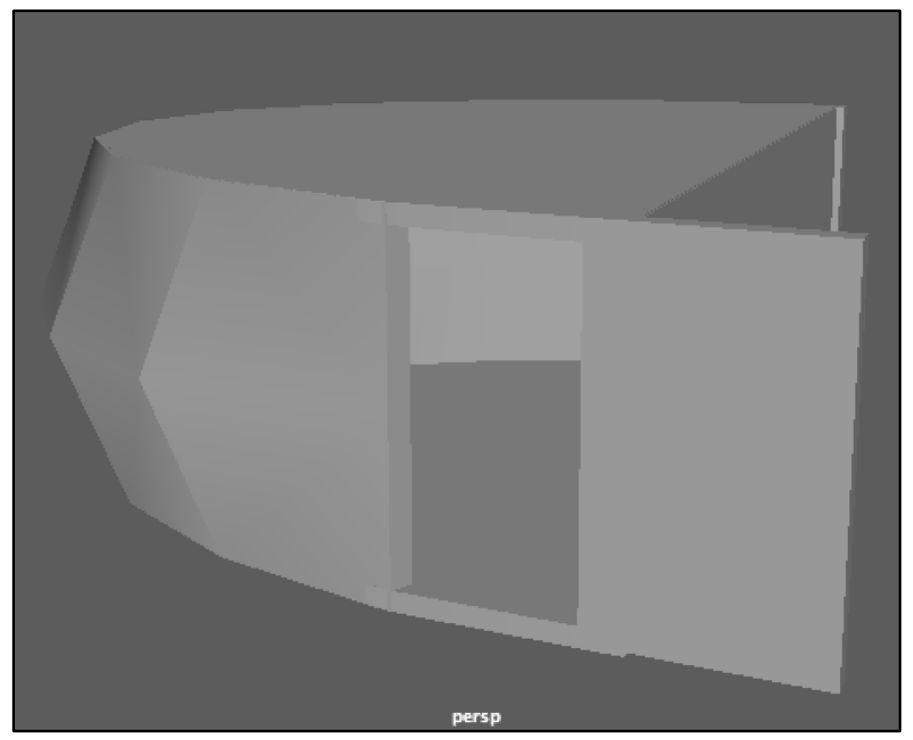

Figure 2. 4: Elevator Model

\subsection{Texturing}

Texturing involves assigning material to the models. Material are either made with color or can be assigned from graphic files. Moreover, there are different types of materials according to where the material is applied. Blin materials are assigned to reflective models e.g. glass, metal, also Lamberts are assigned to non-reflective solid models e.g. walls, wood. 
The bridge is mostly made of red colored material for the iron part. While the street of the bridge is covered with a file material made using Adobe Photoshop CC 2017, to resemble a street. The pavement is textured with cement colored material. See (figure 2.6). all materials are made using the Hypershade window which enables creating different types of materials and connecting them to files or colors. The Hypeshade windows also allows customizing materials to resemble different surfaces with custom reflection, transparency and much more.

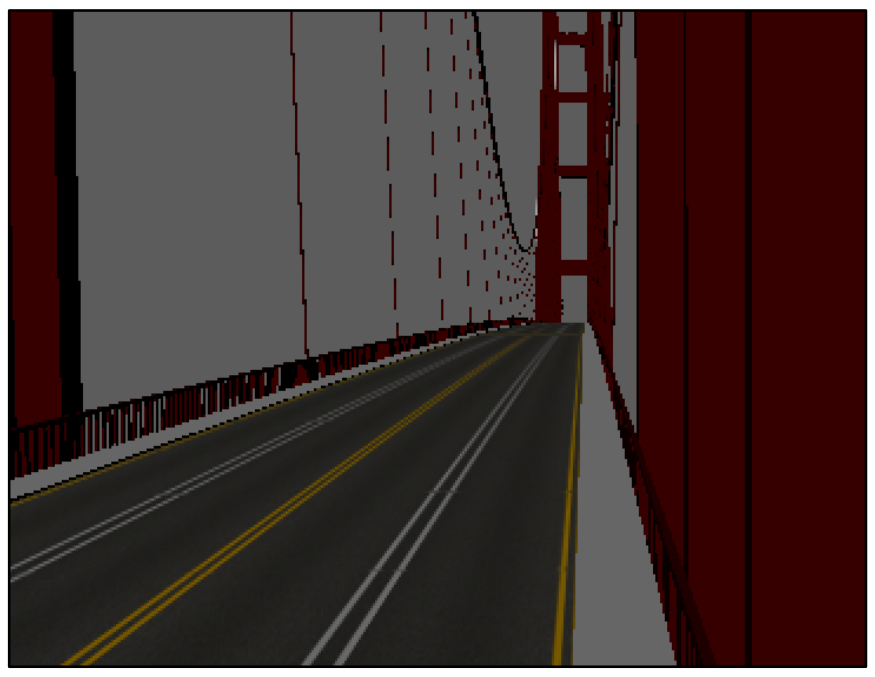

Figure 2. 7: Textured Bridge

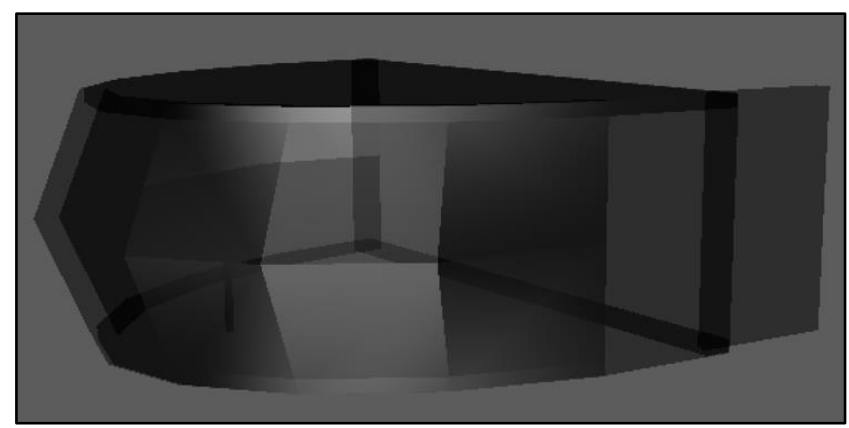

Figure 2. 6: Textured Elevator

Furthermore, the elevator is textured using a Blin material with dark colored glass, the material also incorporated transparency to resemble glass. See (figure 2.7).

\section{Scene}

The virtual reality environment is developed using Unity3d gaming engine. Unity3d is a gaming platform where different $3 \mathrm{~d}$ and $2 \mathrm{~d}$ games are developed. It also allows for custom made models to be used in its environments.

Firstly, the bridge model along with its components and textures are imported to Unity3d. afterwards different technologies are used to complete the virtual reality environment mentioned.

\subsection{Colliders}

Colliders enable adding physical shape to models (Unity Technologies, 2017). Since the bridge is model, without using colliders it is treated as a walk-through object. So colliders must be specified to the model along with the elevator. There are different types of colliders, e.g. cube, cylinder, sphere and mesh collider. Each collider type is specific to a model's shape. In the research, mesh collider is used. A mesh collider adds physics to a non-uniform model. Thus enabled the model to be treated as a real world object with physical collision within the environment.

\subsection{Animation}

Animations can be integrated with objects in unity3d. an animation is usually moving, scaling or rotating an object at runtime (Unity Technologies, 2017). The animations produced in this application were for the elevator door opening and closing, also for the elevator moving up and down the tower. Animations are made using animation clips which are recorded by unity3d at development. Furthermore, animations can be triggered by code or set to auto play.

\subsection{Player Controller}

The player Controller in Unity3d and commonly in gaming is the first person we see though when inside an environment. In other words, the player in the person character inside the environment. In this application Oculus VR player controller is used which is developed by Oculus for virtual reality games and applications. Currently Oculus is the leading platform for VR game development (Oculus VR LLC., 2017). Though the Oculus VR SDK, The OVR player controller is imported which has two cameras positioned to fit the natural eye displacement. With some modifications to the main player controller script, the VR headset pad was used to enable movement of the player inside the environment at sight direction.

\subsection{Raycasting}

Raycasting is the concept of shooting an invisible ray from a point to detect whether any colliders lay in the path of the ray (Unity Technologies, 2017). This concept is very important for virtual reality because unlike computerized games, were there is a keyboard of inputs, VR headsets only two usable inputs, the pad and back button. The pad is used for moving the player controller inside the game resulting in one unused input to trigger the animations inside the environment.

Using rays involves defining a script in C\# or Java, and attaching it to a game object. The script includes an update() method which is called one in each frame of the game. With the script the origin point (center of camera) and destination direction (forward direction) are set to a ray which is casted to return any objects on the direction of the ray. The objects concerned are given tags to be specified so the ray return the specific object insight.

As a result, if the back button is pressed while the elevator door is insight, the door opens and closes using the 
animations assigned. While if the elevator button is insight, the elevator goes up or down if the elevator is down or up respectively, see (figure 3.1). The code responsible for raycasting and triggering animation is illustrated in (figure 3.2).

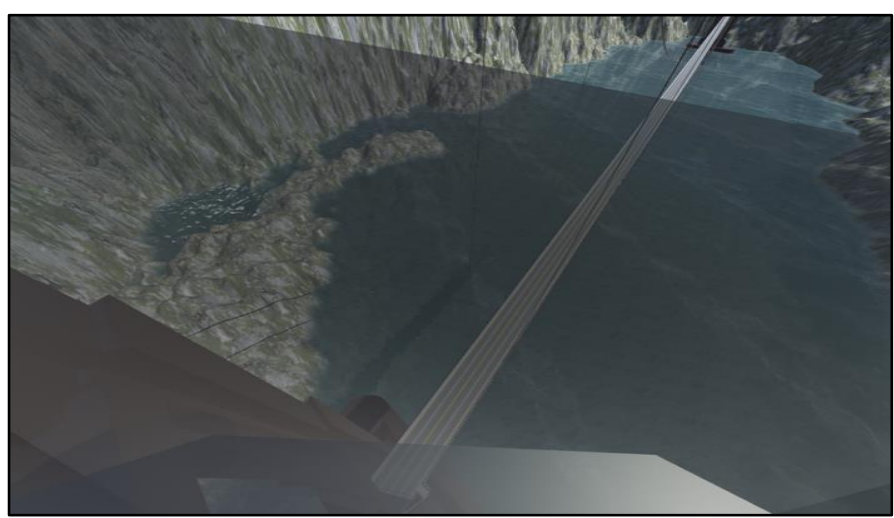

Figure 3. 2: View From Elevator

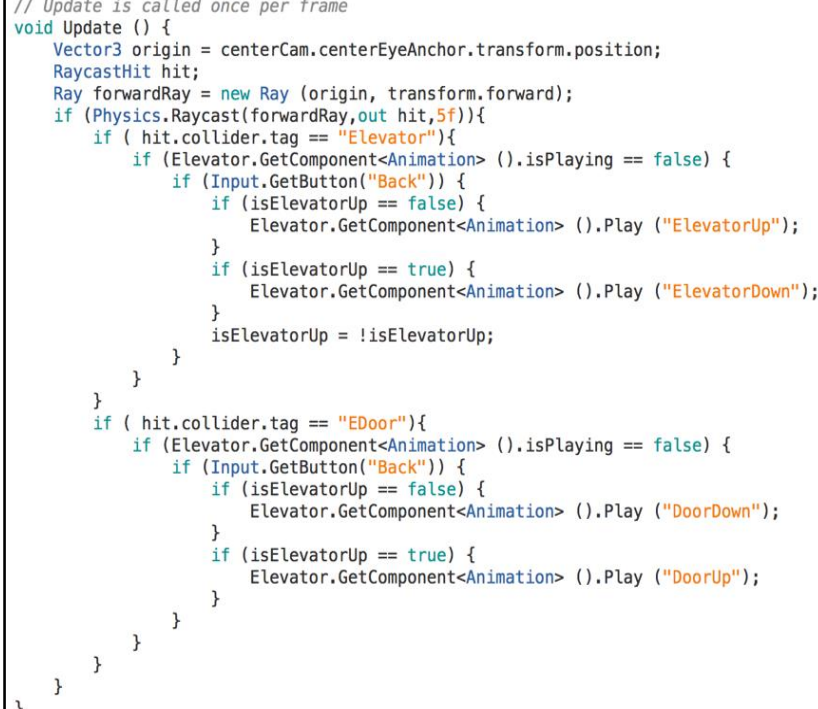

Figure 3. 3: Raycasting and Animation Triggering

\subsection{Spatial 3d Sound Effects}

Spatial sound effect refers to real world $3 \mathrm{~d}$ sound, where the sound at the source is at highest volume and it starts decay as we move further away. The sound clip in this application is the sound of wind. The spatial $3 \mathrm{~d}$ sound effect is set using linear roll-off, where wind sound is strongly heard at the top of the tower. Hence, it continuously decays as the player moves downwards to represent a light breeze at the bridge deck.

\subsection{Environment}

Since any real world environment isn't composed of a single object, it being a building or a tower alone. In this applications, the environment was set by integrating an ocean and mountainous terrains. The ocean has waves along with water reflection in a form of a high sea. Furthermore, the terrains are manipulated to represent mountains where the terrain surface is textured using a grass to rocky mountain texture, see (figure 3.3) and (figure 3.4) for views from the tower and deck within the environment.

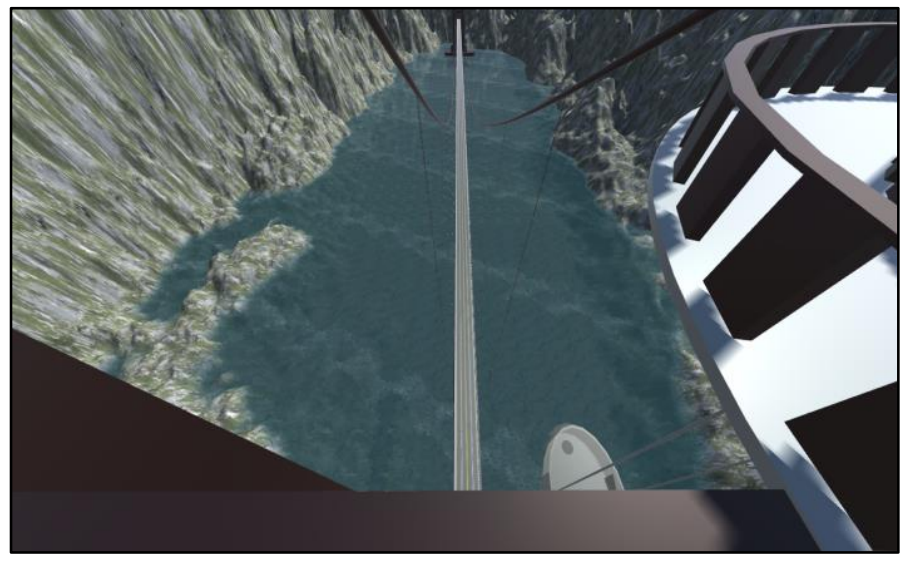

Figure 3. 4: View From Tower

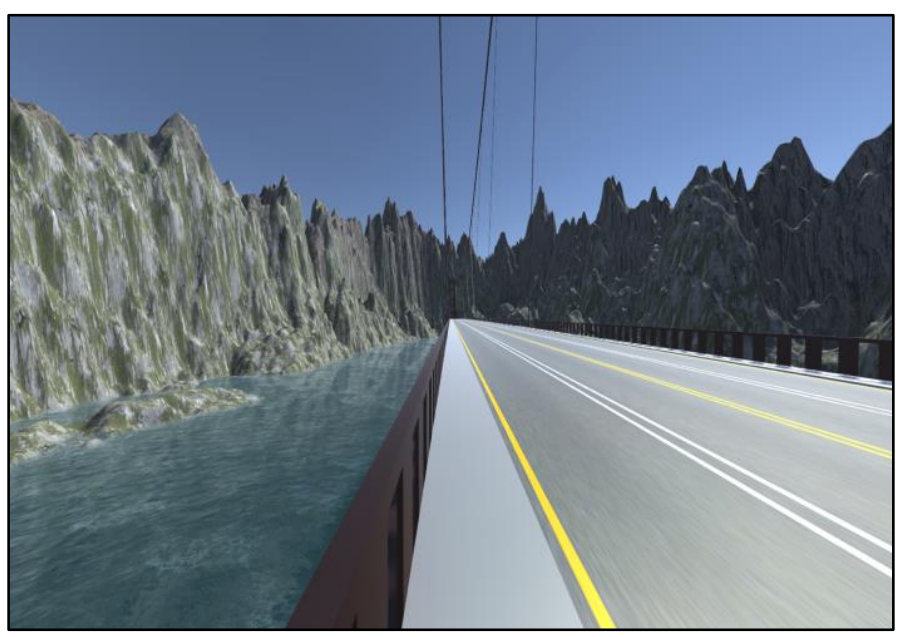

Figure 3. 1: View From Deck

\subsection{Settings and Signing}

VR applications do not build out of the box, when a VR application is developed several settings need to be set. The following settings have been set in this application:

1. Mapping input to detect VR headset controls.

2. Graphics settings to accommodate mobile hardware capabilities.

3. Creating Oculus Signature Files to sign the application, to prevent unauthorized use.

4. Setting the skybox and sunlight source for a more realistic experience.

Further settings are required regarding the build target platform. The devices that currently can run VR applications are Samsung Galaxy 6+ and Samsung Note 4+. So the Android SDK along with Java Virtual Machine JDK must be available and set correctly within the project to perform a build.

\section{Study Results}

Results worldwide indicate the advantages of using VR applications for different phobia treatment. In fact, many 
psychiatric hospitals have adopted this form of treatment to accelerate and enhance patients' recovery from a variety of phobias. Example of such medical complexes are Duke Psychiatry and Behavioral Science hospital in North Carolina, Sydney Phobia Clinic in Australia, The Virtual Reality Medical Center in California, etc. Furthermore, patients have reported much better self control regarding their respective phobias. Also avoiding medical treatment is more natural and comforting.

Other advantages of VR treatment are that patients would avoid the side effects of medicine, which sometimes can be dangerous to other aspects of patients' health, also the cost of medicine sometimes is more that what patients can afford. Moreover, this enables the possibility of undergoing self-administered exposure sessions where psychologists recommend.

\section{Conclusion}

Developing a VR application for a mobile platform isn't a usual task. The constrains imposed by the hardware can sometimes be difficult to overcome. These constrains are absent on most VR desktop platforms which are used for VR applications nowadays. In the development process the following points were concluded:

1. The number of polygons used must be kept as small as possible, the size of polygons used doesn't effect the application's quality. Creating a large geometry from a single polygon is lighter than using two small polygons.

2. Textures are treated like polygons. Using large textures to cover an object is ineffective to rendering time, while using two small textures to the same object will slow down the rendering time.

3. Code execution must be considered which must be less than 3 milliseconds. Writing many lines of code within the update method will slow down the application.

In a desktop VR application, were the headset is connected to a computer with large computing power and graphics capabilities, the previous limitations do not exist. The development process is a usual task of building any other desktop application without baring in mind the processing and graphics capabilities.

\section{REFERENCES}

Autodesk Inc. (2016, May 11). Polygonal Modeling. Retrieved 2 1, 2017, from knowledge.autodesk.com: https://knowledge.autodesk.com/support/maya/le arnexplore/caas/CloudHelp/cloudhelp/2016/ENU/M aya/files/GUID-7941F97A-36E8-47FE-95D171412A3B3017-htm.html

Krijn, M. (2006). Virtual Reality and Specific Phobias. University of Amsterdam. Amsterdam: Cybermind Interactive Nederland and CRLO Displays Limited.
Maatjes, N. C. (2005). The Treatment of Phobias using Virtual Reality. University of Twente, Electrical Engineering, Mathematics and Computer Science. Enschede: University of Twente.

Miloff, A., Lindner, P., Hamilton, W., Reuterskiold, L., Andersson, G., \& Carlbring, P. (2016). Single-session gamified virtual reality exposure therapy for spider phobia vs. traditional exposure therapy: study protocol for a randomized controlled non-inferiority trial. Linköping University. Linköping: Linköping University Electronic Press.

Oculus VR LLC. (2017). Oculus Utilities for Unity. Retrieved May 1, 2017, from Developer.Oculus.com: https://developer.oculus.com/documentation/unity /latest/concepts/unity-utilities-overview/

Parab, T., Pawar, D., \& Chaudhari, A. (2016). A Cost Effective Approach towards Virtual Reality: Phobia Exposure Therapy . International Journal of Advanced Research in Computer and Communication Engineering , 5 (3).

Technologies, U. (n.d.). Raycasting.

Unity Technologies. (2017, March 29). Animation. Retrieved April 1, 2017, from docs.unity3d.com: https://docs.unity3d.com/550/Documentation/Scri ptReference/Animation.html

Unity Technologies. (2017, March 29). Colliders. Retrieved April 1, 2017, from docs.unity3d.com: https://docs.unity3d.com/Manual/CollidersOvervi ew.html

Unity Technologies. (2017). Raycasting. Retrieved April 1, 2017, from Unity3d.com: https://unity3d.com/learn/tutorials/topics/physics /raycasting 